\title{
Glycogen Storage Disease Type IV
}

National Cancer Institute

\section{Source}

National Cancer Institute. Glycogen Storage Disease Type IV. NCI Thesaurus. Code C84737.

A rare inherited type of glycogen storage disease caused by deficiency of amylo-1,4-1,6 transglucosidase. 